\title{
Reovirus enhances cytotoxicity of natural killer cells against colorectal cancer via TLR3 pathway
}

\author{
Shiqi Long ${ }^{1,2+}$, Yangzhuo Gu ${ }^{3 \dagger}$, Yuanyuan An ${ }^{1,2}$, Xiaojin Lin ${ }^{1,2}$, Xiaoqing Chen ${ }^{1,2}$, Xianyao Wang ${ }^{1,2}$, \\ Chunxiang Liao ${ }^{1,2}$, Weiwei Ouyang ${ }^{4}$, Nianxue Wang ${ }^{2}$, Zhixu He He $^{1,5}$ and Xing Zhao ${ }^{1,2,3^{*}}$ (B)
}

\begin{abstract}
Background: Cetuximab has been approved for use for first-line treatment of patients with wild-type KRAS metastatic colorectal cancer (CRC). However, treatment with cetuximab has shown limited efficacy as a CRC monotherapy. In addition, natural killer (NK) cell function is known to be severely attenuated in cancer patients. The goal of this study was to develop a new strategy to enhance antibody-dependent cell-mediated cytotoxicity (ADCC) mediated by NK cells, in combination with cetuximab against CRC cells.
\end{abstract}

Methods: Ex vivo expanded NK cells were stimulated with reovirus, and reovirus-activated NK cells mediated ADCC assay were performed on CRC cells in combination with cetuximab. The synergistic antitumor effects of reovirus-activated NK cells and cetuximab were tested on DLD-1 tumor-bearing mice. Finally, Toll-like receptor 3 (TLR3) knockdown in NK cells, along with chemical blockade of TLR3/dsRNA complex, and inhibition of the TLR3 downstream signaling pathway, were performed to explore the mechanisms by which reovirus enhances NK cell cytotoxicity.

Results: We first confirmed that exposure of NK cells to reovirus enhanced their cytotoxicity in a dose-dependent manner.We then investigated whether reovirus-activated NK cells exposed to cetuximab-bound CRC cells exhibited greater anti-tumor efficacy than either monotherapy. Co-culture of CRC cell lines with reovirus-activated NK cells indicated that NK cytotoxicity was significantly higher in combination with cetuximab, regardless of KRAS mutation status or EGFR expression level. We also found that reovirus activation of NK cells, in conjunction with cetuximab, resulted in significantly stronger anti-tumor efficacy.Finally, TLR3 knockdown, inhibition of TLR3/dsRNA complex or TBK1/IKKE demonstrated that activation of NK cells by reovirus was dependent on TLR3 and its downstream signaling pathway.

Conclusions: This study demonstrated that combination treatment of reovirus-activated NK cells with cetuximab synergistically enhances their anti-tumor cytotoxicity, suggesting a strong candidate strategy for clinical treatment of CRC.

Keywords: Natural killer, Reovirus, Colorectal cancer, Antibody-dependent cell-mediated cytotoxicity, Toll-like receptor 3

*Correspondence: xingzhao@gmc.edu.cn

†Shiqi Long and Yangzhuo Gu contributed equally to this work ${ }^{1}$ Center for Stem Cell and Tissue Engineering Research/School of Basic Medical Sciences, Guizhou Medical University, Guiyang 550004, Guizhou, China

Full list of author information is available at the end of the article

\section{Background}

Colorectal cancer (CRC) is the third most common cancer and the fourth leading cause of cancer-related death worldwide $[1,2]$. In the past two decades, significant advances have been made in the treatment of CRC, including the introduction of oxiplatin, irinotecan, and 
targeted therapeutic monoclonal antibodies $(\mathrm{mAb})$ like cetuximab [3, 4].Cetuximab, a chimeric immunoglobulin G1(IgG1) mAb that targets the epidermal growth factor receptor (EGFR), was approved for use in combination with FOLFIRI for first-line treatment of patients with wild-type $K R A S$ metastatic CRC [5].Cetuximab specifically binds to EGFR,to block the endogenous EGFR ligands and consequently disrupt EGFR-driven signaling, leading to cell cycle arrest and apoptosis. In addition to cetuximab's direct anti-tumor effects, in vivo evidence from both murine models and clinical series suggest that cetuximab exerts antitumor effects in part due to antibody-dependent cell-mediated cytotoxicity (ADCC) [6,7]. ADCC is a mechanism of innate effector cell immunity, initiated by the binding of receptors for the $\mathrm{Fc}$ domain of $\operatorname{IgG}(\mathrm{Fc} \gamma \mathrm{Rs})$. Fc $\gamma$ Rs and their subclasses are expressed on dendritic cells (DCs), monocytes, macrophages, and natural killer (NK) cells. For NK cells, recognition of IgG1 mAbs by FcyRIIIa (CD16) results in enhanced NK cell degranulation, cytokine secretion, and cytotoxicity $[8,9]$. However, despite cetuximab's multiple mechanisms of action, responses to cetuximab are limited: only 1 in 5 patients with metastatic CRC responds to cetuximab [10].New approaches are therefore needed to enhance its efficacy. To this end, increasing the NK cell response to cetuximab therapy could potentially enhance the effects of ADCC in CRC therapy.

NK cells are a crucial target for cancer immunotherapy since they can directly kill tumor cells without prior sensitization or major histocompatibility complex (MHC) restriction. NK cells recognize tumor cells via activating receptors and inhibitory receptors. Activated NK cells directly kill tumor or virus-infected cells through release of cytotoxic granules and proinflammatory cytokines. Recent finding indicate that NK cells exhibit decreased activity, reduction in the proportion of IFN- $\gamma$ secreting NK cells, and predominant CD16 ${ }^{\mathrm{dim} / \text { neg }}$ subpopulation in cancer patients [11, 12]. Additionally, multiple reports found that NK cell function in cancer patients is severely attenuated [13]. Increased expression of programmed cell death protein 1 (PD-1) or T cell immunoglobulin and mucin domain-containing molecule-3(Tim-3) on NK cells suppress NK cell cytotoxicity and correlate with poor prognosis [14-16]. Therefore, extensive studies have investigated different strategies to enhance NK cell cytotoxicity and thereby improve the clinical outcomes of NK cell-based immunotherapies [17-19].

Here, in this work, we sought to develop a new strategy to enhance NK cell cytotoxicity while also improving the effects of cetuximab-mediated ADCC. Our previous research found that reovirus can directly activate fresh NK cells in vitro, and that reovirus-loaded NK cells could deliver reovirus to tumor cells in the presence of neutralizing antibodies (NABs) [20, 21]. Therefore, we hypothesized that the antitumor efficacy of cetuximab could be improved by combination with reovirus activation of NK cells. In this strategy, NK cells are first activated with reovirus, followed by exposure of the reovirus-activated NK cells to cetuximab-bound CRC cells. Using multiple CRC cell lines with different KRAS mutation status and EGFR expression levels we found that reovirus directly enhanced NK cell cytotoxicity,and that, in combination with cetuximab, reovirus-activated NK cells exhibited increased CRC cell killing in vitro,regardless of KRAS mutation or EGFR expression.Then using human colorectal tumor xenograft models, we also observed that reovirus activation of NK cells, in conjunction with cetuximab, provided significantly greater anti-tumor effects than either monotherapy. Finally, using Toll-like receptor 3 (TLR3) knockdown NK cells,chemical blockade of TLR3/double-stranded RNA (dsRNA) complex,and chemical inhibition of the TLR3 downstream pathway, we further determined that reovirus activation of NK cells is mediated in a TLR3 signaling pathway-dependent manner.These findings of synergistically enhanced NK cell anti-tumor activity provide a viable framework for improvement of clinical strategies against CRC.

\section{Materials and methods \\ Cells, virus, and reagents}

The murine fibroblastic cell line L929, the EGFRexpression CRC cell lines DLD-1 (KRAS-mutant, EGFR medium), Caco-2 (KRAS-WT, EGFR high), and HT-29 (KRAS-WT, EGFR low) were obtained from the China Center for Type Culture Collection (CCTCC) [6]. All cell lines were cultured in MEM and RPMI-1640 (Hyclone) supplemented with $10 \%$ fetal bovine serum (FBS, Gibco), $1 \%(\mathrm{v} / \mathrm{v})$ glutamine (Gibco) and $1 \%(\mathrm{v} / \mathrm{v})$ penicillin/streptomycin (Hyclone). All cells were maintained at $37^{\circ} \mathrm{C}, 5 \%$ $\mathrm{CO} 2$ in a humidified atmosphere.Reovirus type 3 Dearing strain was obtained from ATCC (VR-824). Reovirus was propagated in L929 cells, and titrated with a standard plaque assay protocol for evaluating L929 cells. To generate UV-inactivated reovirus. Reovirus in PBS were exposed to UV (shortwave $254 \mathrm{~nm}$ ) for $30 \mathrm{~min}$. The UVinduced loss of reoviral replicability was confirmed with a L929 cell viability assay.Live and UV-inactivated reovirus were stored at $-80{ }^{\circ} \mathrm{C}$ until use. Cetuximab (murinehuman chimeric anti-EGFR, IgG1) was obtained from Bristol-Myers Squibb. Control IgG1 purchased from Sigma-Aldrich.Bx795 (Sigma-Aldrich) was used to inhibit TBK/IKKe,TLR3/dsRNA complex inhibitor was purchased from Calbiochem. Polyinosinic:polycytidylic acid [Poly (I:C)] HMW was obtained from InvivoGen. 


\section{Ex vivo NK cell expansion}

Peripheral blood mononuclear cells (PBMCs) from healthy donor blood were isolated by means of standard density gradient centrifugation with the use of FicollPaque Plus (GE healthcare).NK cells were enriched from PBMCs as mentioned below. Briefly, $5 \times 10^{6}$ PBMCs were cultured in GT-T551 H3 medium (Takara Bio Inc.) supplemented with $1 \%$ autologous serum and $200 \mathrm{IU} / \mathrm{mL}$ of rhIL-2 (PeproTech), and co-cultured with $5 \times 10^{6}$ irradiated inactivated K562-mbIL-21 feeder cells (Lifeark). Media were changed every $3-4$ days with a weekly addition of K562-mbIL-21.NK cells were harvested after 14-16 days of incubation and assessed for purity (NK cells defined as $\mathrm{CD}^{-} \mathrm{CD} 14^{-} \mathrm{CD} 56^{+}$) via flow cytometry (>95\% purity was achieved). NK cells were aliquoted and cryopreserved in liquid nitrogen until use. To minimize phenotypic changes, all NK cells were used within two months of the initial freezing date.

\section{Cytotoxicity assay}

The cytotoxic activity of NK cells was assessed using Cell Counting Kit-8 (CCK-8, Dojindo).Briefly, NK cells were thawed in a $37{ }^{\circ} \mathrm{C}$ water bath and washed twice in GT-T551 H3 medium before use. NK cells were maintained in GT-T551 H3 medium for at least $2 \mathrm{~h}$, before they were resuspended at $2 \times 10^{6}$ cells $/ \mathrm{mL}$ and incubated with 10 multiplicity of infection (MOI) reovirus or $1 \mu \mathrm{g} /$ mL Poly(I:C) complexed with Lipofectamine 3000 (Invitrogen) for $12 \mathrm{~h}$ at $37{ }^{\circ} \mathrm{C}$ and $5 \% \mathrm{CO} 2$. After $12 \mathrm{~h}$ of pretreatment, NK cells were incubated with target tumor cells (DLD-1, Caco-2, and HT-29) at 5:1 E:T ratios for $4 \mathrm{~h}$ in U-bottom 96-well plates at $37^{\circ} \mathrm{C}, 5 \% \mathrm{CO}$. For ADCC assay, target tumor cells were incubated with $1 \mu \mathrm{g} / \mathrm{mL}$ cetuximab or control antibody IgG1 for $1 \mathrm{~h}$. Following incubation, the cytotoxicity assay was performed. For some blocking experiments, reovirus or Poly(I:C) acti-

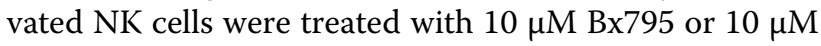
TLR3/dsRNA complex inhibitor for $12 \mathrm{~h}$ at $37^{\circ} \mathrm{C}$ and $5 \%$ $\mathrm{CO} 2$ prior to the addition of NK cells. After a 4-h incubation, $10 \mu \mathrm{L}$ of supernatant was collected from each well and the absorbance at $450 \mathrm{~nm}$ of each well was measured according to the manufacturer's instructions. All experiments were done in triplicate.

\section{siRNA-mediated gene knockdown}

To knock down the expression of TLR3, NK cells were transfected with siRNA against TLR3 (siTLR3 no. 337 5'-GCUUGGAUGUAGGAUUUAATT-3' \& 5'-UUA AAUCCUACAUCCAAGCTT- $3^{\prime}$, Gene Pharma) or random control siRNA (5'-UUCUCCGAACGUGUCACG UTT-3'. \& 5'-ACGUGACACGUUCGGAGAATT-3', Gene Pharma) using the Lipofectamine 3000 transfection reagent (Invitrogen, USA) for the indicated durations. The TLR3 knockdown efficacy was confirmed by qPCR and Western Blotting analysis. Following a 48-h incubation, NK cells were incubated with $10 \mathrm{MOI}$ reovirus or $1 \mu \mathrm{g} / \mathrm{mL}$ Poly(I:C) for $12 \mathrm{~h}$ prior to coculture with DLD-1 cells.

\section{Western blotting}

Cell lysates were prepared in cell lysis buffer. Extracts were analyzed by SDS-PAGE (Invitrogen) and western blot. The following antibodies were used at specified concentrations for immunoblots:TLR3 (Clone D10F10; 1:1,000; Cell Signaling Technology; \#6961) and $\beta$-actin (Clone 5B7; 1:5,000;ImmunoWay; YM3028). The secondary antibody was HRP-conjugated donkey anti-rabbit IgG $(\mathrm{H}+\mathrm{L})(1: 10,000$; Biodragon; BF03008X). Antigenantibody complexes were visualized by enhanced chemiluminescence (Bio-Rad). Western blots were quantified using ImageJ software (National Institutes of Health).

\section{Real-time quantitative PCR (qPCR)}

Total RNA was extracted from cells using Trizol reagent (TaKaRa) and reverse-transcribed into cDNA using iScript cDNA Synthesis Kit (BioRad). The mRNA expression of GZMH, GZMM,PRF1,TNF, TLR3, T3D, and $\beta$-actin was quantified by SYBR Green real-time PCR. Real-time PCR amplifications were performed using Premix Ex TaqTM (TaKaRa) on a Bio-Rad CFX96 system. The PCR primers used in this study (Additional file 1: Table S1) were purchased from Sangon Biotech (Shanghai) Co., Ltd. Amplifications were performed by activation of Hot Start DNA polymerase at $95{ }^{\circ} \mathrm{C}$ for $30 \mathrm{~s}$, followed by 40 cycles at $95^{\circ} \mathrm{C}$ for $5 \mathrm{~s}$ and $60^{\circ} \mathrm{C}$ for $45 \mathrm{~s}$. Data were calculated using Bio-Rad CFX manager software v.2.1 and the $\Delta \Delta C T$ method, and expressed as relative quantities after $\beta$-actin normalization.

\section{ELISA}

For certain experiments, the supernatants were collected and stored at $-80{ }^{\circ} \mathrm{C}$ until the time of use. The levels of IFN- $\gamma$, TNF- $\alpha$, and perforin in supernatants were determined with the Human IFN-gamma Quantikine ELISA Kit (R\&D Systems), the Human TNF-alpha Quantikine ELISA Kit (R\&D Systems) and the Perforin human ELISA Kit (Abcam) according to the manufacturers' protocols.

\section{Tumor transplantation and therapy}

All animal experiments performed during this study were governed by the approval of the Ethics Committee at Guizhou Medical University, Guiyang, Guizhou Province, China. Five-to six-week-old female athymic BALB/c nu/nu mice were obtained from Beijing HFK Bio-Technology Co., Ltd and maintained at the Animal 
Experimental Center of Guizhou Medical University. DLD-1 cells $\left(2 \times 10^{6}\right)$ were implanted s.c. on the left flank. After tumors reached $\sim 50 \mathrm{~mm}^{3}$, mice were divided into four groups on day 10. Mice received an intravenous injection of phosphate-buffered saline (PBS, $100 \mu \mathrm{L}$ ), or NK cells pretreated with $10 \mathrm{MOI}$ reovirus monotherapy (Reo-NK, $1 \times 10^{7}$ cells), or were intraperitoneally treated with cetuximab at $200 \mu \mathrm{g}$ monotherapy, or sequential intravenous injection of $1 \times 10^{7}$ Reo-NK $12 \mathrm{~h}$ prior to being intraperitoneally treated with cetuximab dosed as in the monotherapy group. Animals were dosed for 3 weeks. Tumor size was measured with a caliper twice a week and calculated as length $\times$ width $\times$ height. Mice were sacrificed at day 26 . This in vivo model was piloted with three mice per group and repeated with five mice per group. Tumors, liver and kidney were collected from each mouse, and then the tumors were used for qPCR analysis. The section of liver and kidney were stained by Hematoxylin and Eosin $(\mathrm{H} \& \mathrm{E})$ according to manufacturer's instruction.

\section{Flow cytometry immunophenotyping}

For NK cell staining, we used the following antibodies: anti-CD3 (clone: UCHT1), anti-CD56 (clone:MEM-188), anti-CD69 (clone: FN50) all from BioLegend; and antiTLR3 (clone: TLR3.7, Invitrogen). FITC-IgG2b, $\kappa$ isotype (BD Biosciences) was used as an isotype control. All cell samples were pre-incubated with anti-CD16/32 antibody to block non-specific binding. Following Fc-receptor blocking. Cells were washed once with cold FACS buffer (PBS supplemented with $2 \% \mathrm{FBS}$ and $0.1 \%$ sodium azide). Cells were stained $30 \mathrm{~min}$ at room temperature with the surface-staining antibodies.TLR-3 intracellular staining was performed using the Cytofix/Cytoperm Kit (BD Biosciences). Stained cells were collected on a FC500 flow cytometer (Beckman Coulter Inc.), and data were analyzed using Flowjo software.

\section{Statistical analyses}

Statistical analyses were performed using GraphPad Prism version 8 software. For normally distributed variables, comparisons between two groups were performed by the parametric Student's t-test; multiple group comparisons were carried out by one-way analysis of variance (ANOVA). Significant differences were defined as: * $p<0.05$, "** $p<0.01$, ${ }^{* * * *} p<0.001$.

\section{Results}

\section{Stimulation with reovirus enhances NK cell-mediated cytotoxicity}

Given previous findings that NK cells can lyse tumor cells without prior exposure, and that NK cell cytotoxicity can be enhanced through pre-treatment with Poly(I:C), IL-2, or IL-15 [22, 23], we therefore used ex vivo expanded NK cells to evaluate their cytotoxicity following reovirus mediated activation.The results of NK cell cytotoxicity analysis showed that stimulation with different titers of reovirus and subsequent co-culture with DLD-1 target cells at a 5:1 effector-to-target-cell (E:T) ratio enhanced NK cell cytotoxicity in a dose-dependent manner (Fig. 1a). As CD69 is a marker for NK cell activation, we also assessed their activation through flow cytometry assays, which confirmed that CD69 was up-regulated in response to reovirus stimulation (Fig. 1b, c).Perforin (PRF1) and granzymes (such as GZMA, GZMB, GZMM and GZMH) represent two distinct categories of cytotoxic proteins released by NK cells. In addition, TNF- $\alpha$ has been shown to work in concert with IFN- $\gamma$ to promote NK cell cytotoxicity. We applied qPCR analysis, which showed that the expression of GZMH, GZMM, PRF1, and TNF were all up-regulated in the NK cells treated with $10 \mathrm{MOI}$ of reovirus for $12 \mathrm{~h}$ (Fig. 1d).

Poly(I:C), a synthetic ligand mimicking viral dsRNA, was shown in previous studies to increase the cytotoxicity of NK cells $[24,25]$. In light of these findings, we next tested the effects of lipofectamine-packaged Poly(I:C) on NK cells. NK cells were treated with $10 \mathrm{MOI}$ reovirus or $1 \mu \mathrm{g} / \mathrm{mL}$ Poly(I:C) for $12 \mathrm{~h}$ prior to co-culture with DLD-1 cells. We found that the cytotoxicity of NK cells was significantly increased following treatment with 10 MOI reovirus $(60.10 \pm 3.67, \mathrm{n}=3, p<0.05)$ or lipofectamine-Poly(I:C) $(65.38 \pm 5.05, \mathrm{n}=3, p<0.01)$ compared to that of un-activated control cells $(45.84 \pm 0.72$, $\mathrm{n}=3$ ) (Fig. 1e). As reovirus has a double stranded RNA genome, which suggests that NK cells are activated by reovirus in a manner similar to that of Poly(I:C). Taken together, these results indicate that NK cell cytotoxicity can be enhanced through exposure to reovirus.

\section{Stimulation reovirus-activated NK cells enhance cetuximab-mediated ADCC against CRC cells}

Previous studies have reported that cetuximab can be used as a bridge to connect NK cells and CRC cells to trigger ADCC, thus killing colorectal cells $[8,26]$. Therefore, promoting NK cytotoxicity can enhance the effects of ADCC mediated by cetuximab. Moreover, the ability of reovirus to enhance NK cytotoxicity makes it an ideal candidate for combination therapeutic strategies, such as with cetuximab.To examine whether reovirus activation of NK cells also enhances ADCC against EGFR-expressing CRC cells, we used ex vivo expanded NK cells activated with $10 \mathrm{MOI}$ reovirus for $12 \mathrm{~h}$ to perform ADCC assays on colorectal cancer cells also treated with cetuximab. DLD-1 (KRAS-mutant, EGFR medium), Caco-2 (KRAS-WT, EGFR high), and HT-29 (KRAS-WT, EGFR low) were incubated with NK cells at a 5:1 E:T ratio [6], 

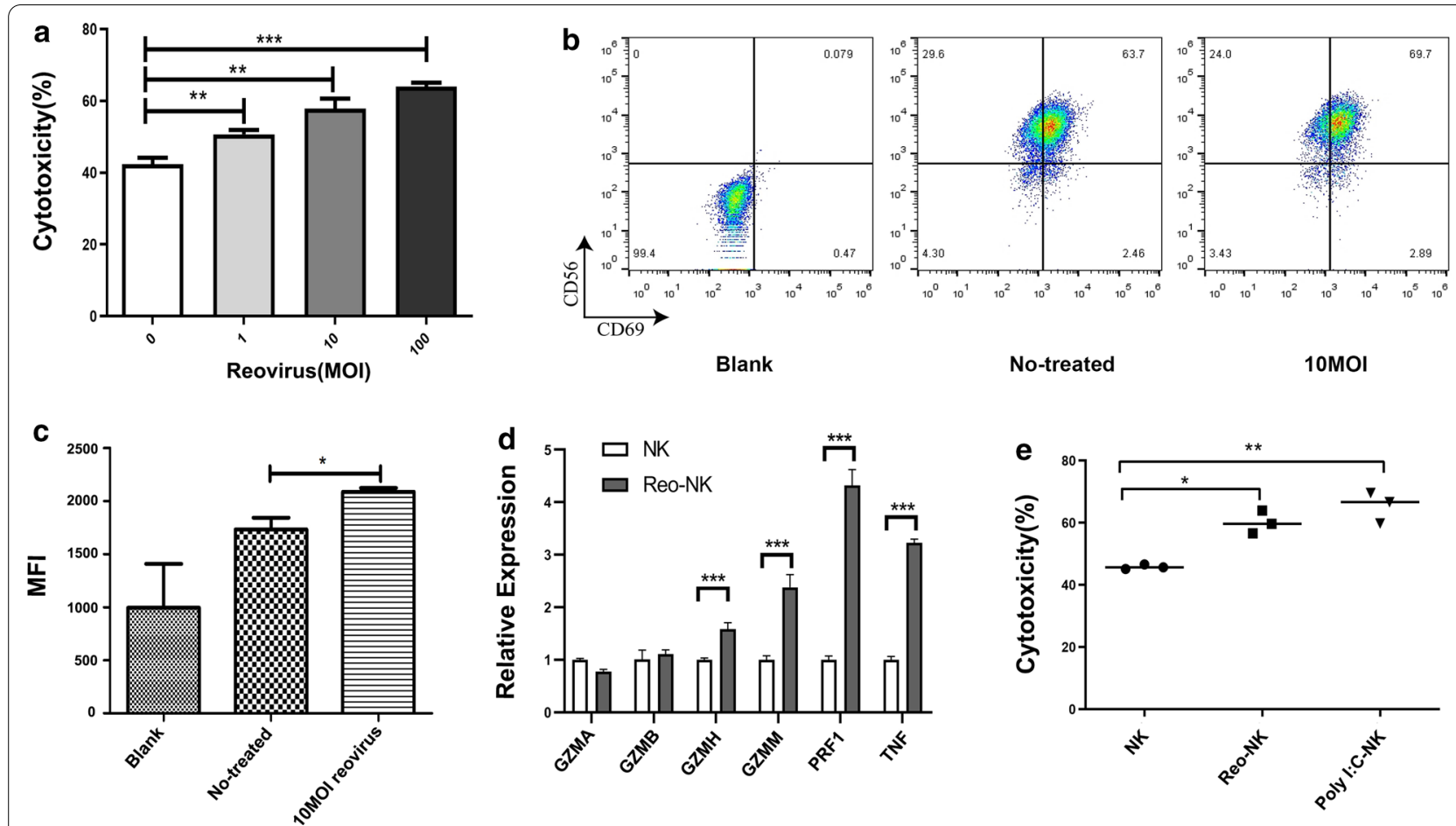

Fig. 1 Effects of reovirus on NK cells. a NK cells were incubated in the absence or presence of 1, 10, or $100 \mathrm{MOI}$ reovirus at $37^{\circ} \mathrm{C}$ for $12 \mathrm{~h}$. DLD-1 cells were used as target cells. Cytotoxic activity was measured at $4 \mathrm{~h}$ by CCK-8 assays performed on target cells at an E:T ratio of 5:1. All samples were assayed in triplicate and these data are representative of three independent experiments. $\left.{ }^{* *} p<0.01,{ }^{* * *} p<0.001\right)$. b Representative flow cytometry plots showing the expression of CD69 on NK cells stimulated or not with $10 \mathrm{MOI}$ reovirus for $12 \mathrm{~h}$. c Compiled data (mean \pm SEM) from three independent experiments showing mean fluorescence intensity (MFI) for CD69, d qPCR evaluation of the relative expression of GZMA, GZMB, GZMH, GZMM, PRF1, and TNF in NK cells after $12 \mathrm{~h}$ exposure to $10 \mathrm{MOI}$ reovirus or medium alone (control; NK).qPCR experiments were performed twice with three technical replicates per sample. Data are means $\pm S D\left({ }^{* *} p<0.001\right)$. e NK cells were incubated at $1 \times 10^{6}$ cells $/ \mathrm{mL}$ for $12 \mathrm{~h}$ in medium alone or $10 \mathrm{MOI}$ reovirus; NK cells were transfected with $1 \mu \mathrm{g} / \mathrm{mL}$ Poly(l:C) by Lipofectamine and also cultured for $12 \mathrm{~h}$. The NK cells stimulated under the various conditions were then co-cultured with DLD-1 cells for $4 \mathrm{~h}$ at a 5:1 E:T ratio. NK cell cytotoxicity was determined by CCK-8 assay. Data shown above are from a representative assay selected from three independent experiments. $\left({ }^{*} p<0.05,{ }^{* *} p<0.01\right)$

and we found that NK cells could efficiently kill DLD-1 (Fig. 2a), as well as Caco-2 and HT-29 (Fig. 2b) target cells. Furthermore,NK cell cytotoxicity was increased after activation with $10 \mathrm{MOI}$ reovirus, and the reovirusactivated NK cells showed enhanced ADCC in combination with cetuximab treatment, indicated by decreasing tumor cell viability. Colorectal cancer cells were all highly sensitive to killing by reovirus-activated NK cells regardless of KRAS genotype or EGFR expression level, and reovirus further enhanced NK-cell cytotoxicity against cetuximab-coated colorectal cancer cells (Fig. 2a, b).

As NK cell cytotoxicity is mediated by the main effector molecule perforin, which creates pores on the target cells to facilitate entry of apoptosis-inducing granzymes, we therefore hypothesized that one mechanism by which reovirus might enhance and prime NK cell function is by increasing perforin release from NK cells. Detection by ELISA assays supported this hypothesis, and indeed, reovirus-activated NK cells released significantly higher levels of perforin with cetuximab against DLD-1 and HT-29 cells (Fig. 2c and d). Taken together, these data indicate that stimulation with reovirus enhances NK cell-mediated ADCC against colorectal cancer cells, and synergistically so in combination treatment with cetuximab.

\section{Reovirus-activated NK cells enhance the antitumor activity} of cetuximab against KRAS-mutant tumors in vivo

Since the combination of cetuximab with reovirus-activated NK cells together led to increased lysis of colorectal cancer cells in vitro, we next assessed the efficacy of this combination therapy using human colorectal tumor xenograft models. Expanded NK cells were activated with 10 MOI reovirus (Reo-NK), followed by transfer into tumor-bearing mice,or intraperitoneal treatment with $200 \mu \mathrm{g}$ cetuximab per mouse 1 day after transfer, or a combination of reovirus-activated NK cells and cetuximab. Animals were dosed for three weeks (Fig. 3a). The xenograft tumor growth curves showed restriction in tumor growth evidently following the third treatment. By day 26, we observed that the combination therapy 

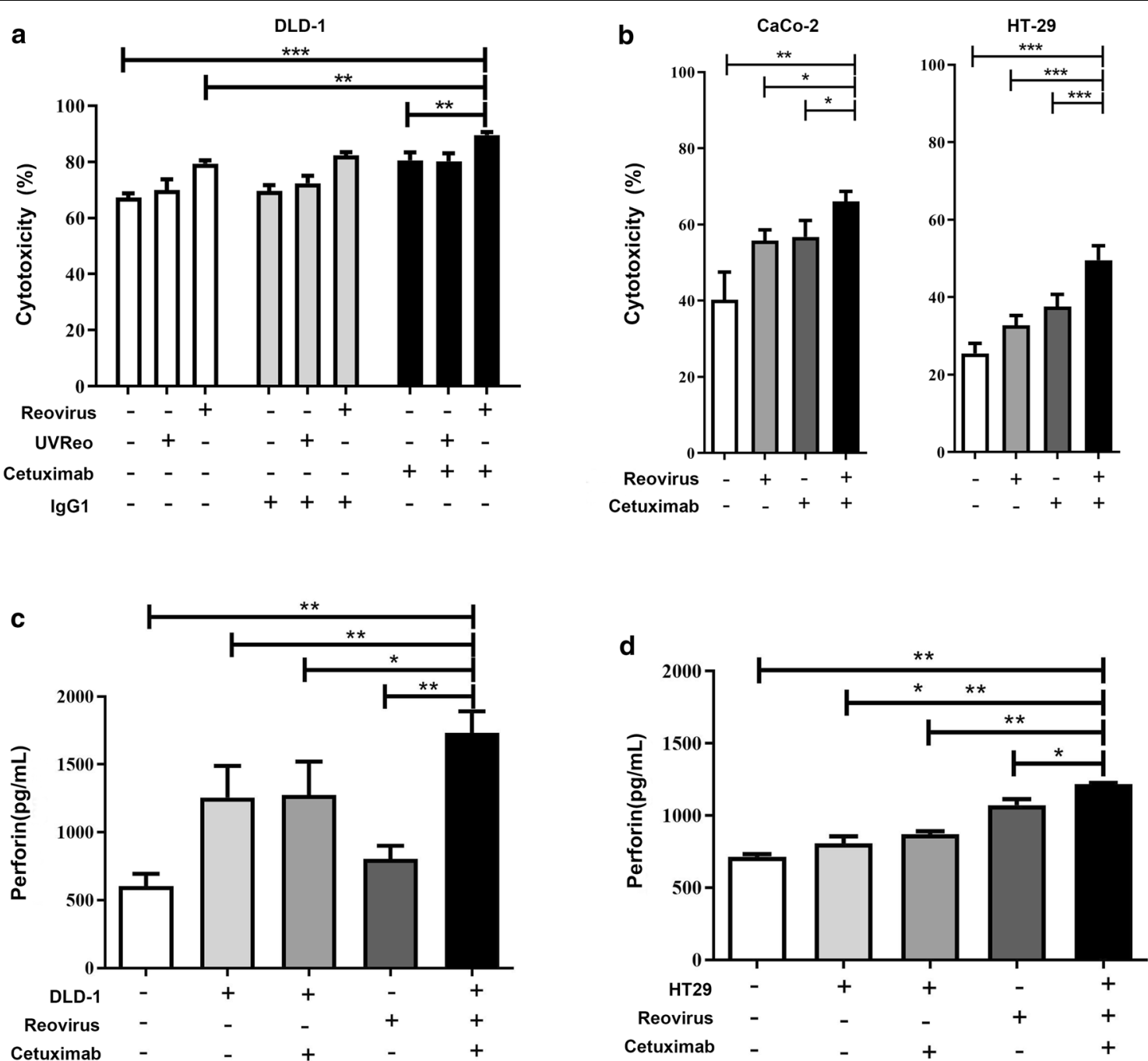

Fig. 2 Reovirus-activated NK cells exhibit enhanced ADCC-mediated killing of colorectal cancer cells in combination with cetuximab. NK cells, as effectors, were activated with $10 \mathrm{MOI}$ reovirus or UV-inactivated reovirus at $37^{\circ} \mathrm{C}$ for $12 \mathrm{~h}$. a DLD-1, or $\mathbf{b}$ Caco- 2 and HT2 29 cells were used as target cells. Cetuximab and isotype control lgG1 were applied at $1 \mu \mathrm{g} / \mathrm{mL}$ and cytotoxic activity was measured by CCK-8 assay at an E:T $=5: 1$ ratio after $4 \mathrm{~h}$ of co-culture. ${ }^{*} p<0.05,{ }^{* *} p<0.01,{ }^{* * *} p<0.001$. Data represent means \pm SD from at least 3 independent experiments. $\mathbf{c}$, $\mathbf{d}$ Levels of perforin secretion from reovirus-activated NK cells against DLD-1 or HT29 combined with cetuximab were determined by ELISA

significantly suppressed tumor growth $(227.8 \pm 65.76$ $\left.\mathrm{mm}^{3}, p<0.05\right)$, although reovirus- activated NK cells $\left(371.9 \pm 91.12 \mathrm{~mm}^{3}\right)$ and cetuximab $\left(348.1 \pm 86.30 \mathrm{~mm}^{3}\right)$ also both showed inhibitory effects on tumor growth (Fig. 3b) compared with the PBS control $(444.8 \pm 204.1$ $\mathrm{mm}^{3}$ ). We also evaluated the tumor weight for each group, and the results confirmed that the combination therapy significantly inhibited tumor growth in nude mice over that of individual treatments (Fig. 3c). Given that Reo-NK and cetuximab combined therapy resulted in strong antitumor effects against localized DLD-1 tumors, we next sought to determine whether the efficacy was related to tumor cell lysis by the reovirus. qPCR results showed significantly detectable reovirus replication in the tumor tissues treated with both cetuximab and reovirus-activated NK cells, whereas the virus not detectable in tumors treated reovirus-activated NK cells alone (Fig. 3d, p<0.001). These results suggested that cetuximab activity enabled viral replication and lysis in tumor cells, potentially by mediating viral transfer from NK cells to tumor cells.

The potential side effects or toxicity of combination therapy are always a great concern in clinical application. The side effect study of reovirus-activated NK cell combined with cetuximab was conducted by $H \& E$ staining. Liver and kidney were collected on day 26 and subjected 


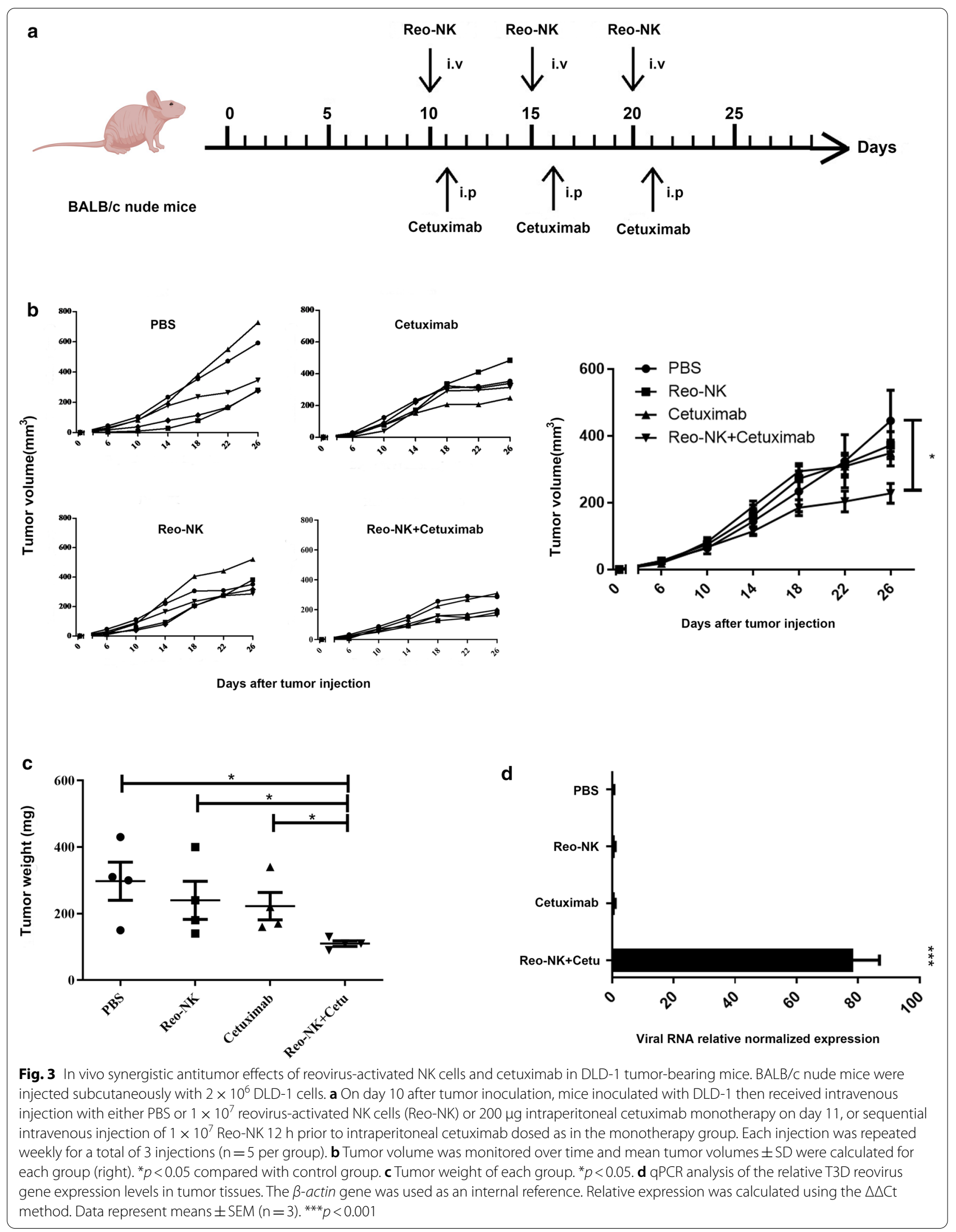




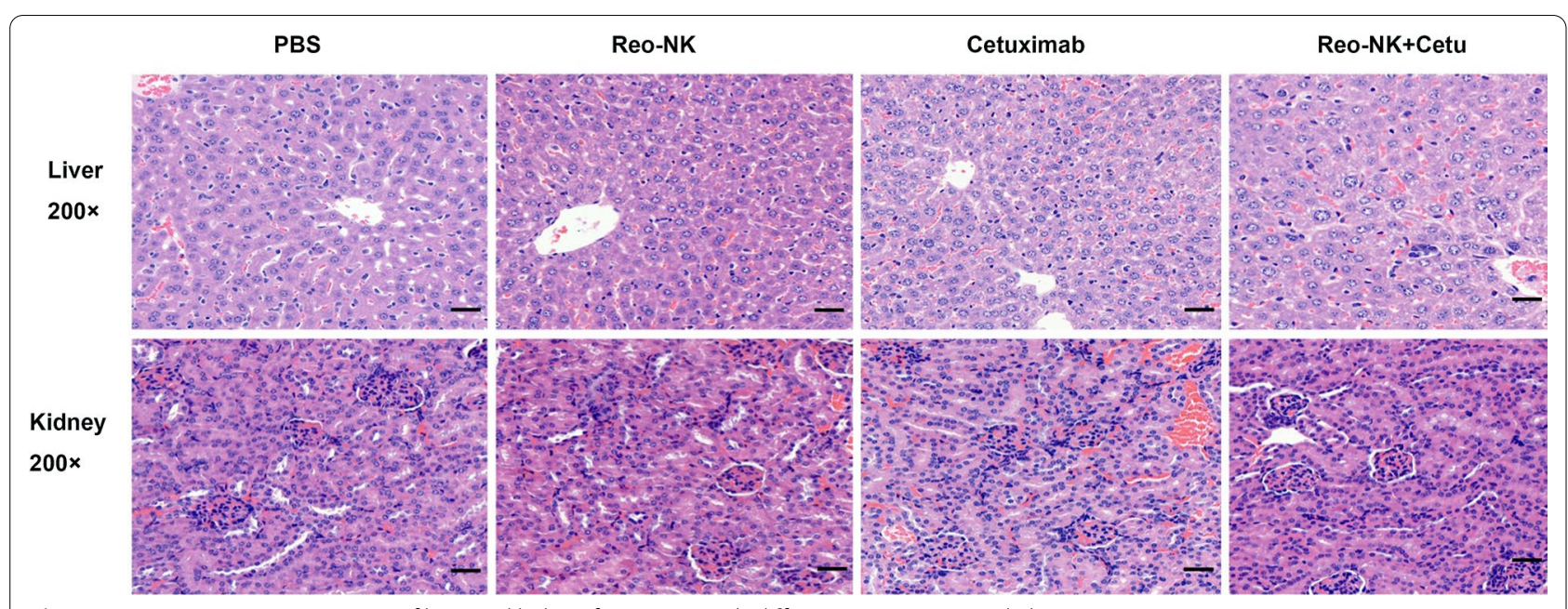

Fig. 4 Representative H\&E staining of liver and kidney from mice with different treatments. Scale bars, 50 um

to H\&E staining. The results indicated that no toxic pathological changes in mice's liver and kidney (Fig. 4).

\section{TLR3 senses reovirus in NK cells}

To examine the mechanisms by which reovirus enhances NK cell cytotoxicity.We first determined which receptor participated in reovirus-induced NK cell activation. Based on our previous evidence that NK cells were activated by reovirus in a mechanism apparently similar to that of Poly(I:C), we therefore focused on the reovirus dsRNA genome. TLR3 is anchored to the endosomal membrane to recognize extracytoplasmic dsRNA, such as Poly(I:C).We reasoned that reovirus could infect NK cells, thereby delivering dsRNA to the cell interior, and subsequently activating TLR3, similar to lipofectaminepacked Poly(I:C). To test this hypothesis, we first determined mRNA and protein expression levels of TLR3 in NK cells, and found that TLR3 mRNA and protein levels were both elevated in NK cells after reovirus administration (Fig. 5a and b). The expression of TLR3 was also confirmed by intracellular staining and flow cytometry, which revealed a slight increase in TLR3 in reovirustreated NK cells (Additional file 2: Figure S1), a finding which was also consistent with the results of Western blotting.

To further confirm that TLR3 may participate in reovirus-induced $\mathrm{NK}$-cell activation, we used siRNA targeting to knockdown TLR3 expression in reovirus-activated NK cells (Additional file 3: Figure S2). We then found that TLR3 knockdown significantly reversed the reovirus- or Poly(I:C)-induced cytotoxicity (Fig. 5c), while negative control NK cells remained responsive to reovirus activation. We then used TLR3/
dsRNA complex inhibitor, a competitive inhibitor of dsRNA binding to TLR3, to confirm the contribution of TLR3/dsRNA binding in reovirus-mediated NK cell activation. The results of this experiment showed that the inhibitor significantly decreased both reovirus- and Poly(I:C)-enhanced cytotoxicity (Fig. 5d). In addition, release of TNF- $\alpha$ and IFN- $\gamma$, well-established potent anti-tumor factors, was also significantly induced after $24 \mathrm{~h}$ of stimulation with $10 \mathrm{MOI}$ reovirus or Poly(I:C), but decreased after treatment with TLR3/dsRNA complex inhibitor (Fig. 5e).Together, these results indicate that TLR3 serves as the primary receptor for modulating the activation and function of NK cells by reovirus.

\section{TBK/IKKe mediates NK cell response to reovirus}

To further clarify the pathway for TLR3-dependent reovirus activation of $\mathrm{NK}$ cells, we examined the effect of blocking TBK1/IKKe, a downstream effector molecule in the TLR3 signaling pathway. To this end, NK cells were incubated for $12 \mathrm{~h}$ with $10 \mathrm{MOI}$ reovirus in the presence of Bx795, a small molecule inhibitor of TBK1/IKKe. We found that Bx795 inhibited reovirus and Poly(I:C) enhancement of NK cell cytotoxicity (Fig. 6a). In addition, CD69 expression on NK cells was similarly decreased after Bx795 treatment (Fig. 6b, c). Furthermore, we observed that perforin secretion was increased with exposure to 10 MOI reovirus or $1 \mu \mathrm{g} /$ mL Poly(I:C), but decreased with Bx795 treatment, and that IFN- $\gamma$ secretion from NK cells was also reduced after TBK1/IKKe blockade (Fig. 6d). These results cumulatively show that TLR3-dependent response to reovirus is mediated by the TBK $1 / \mathrm{IKK} \varepsilon$ signaling. 

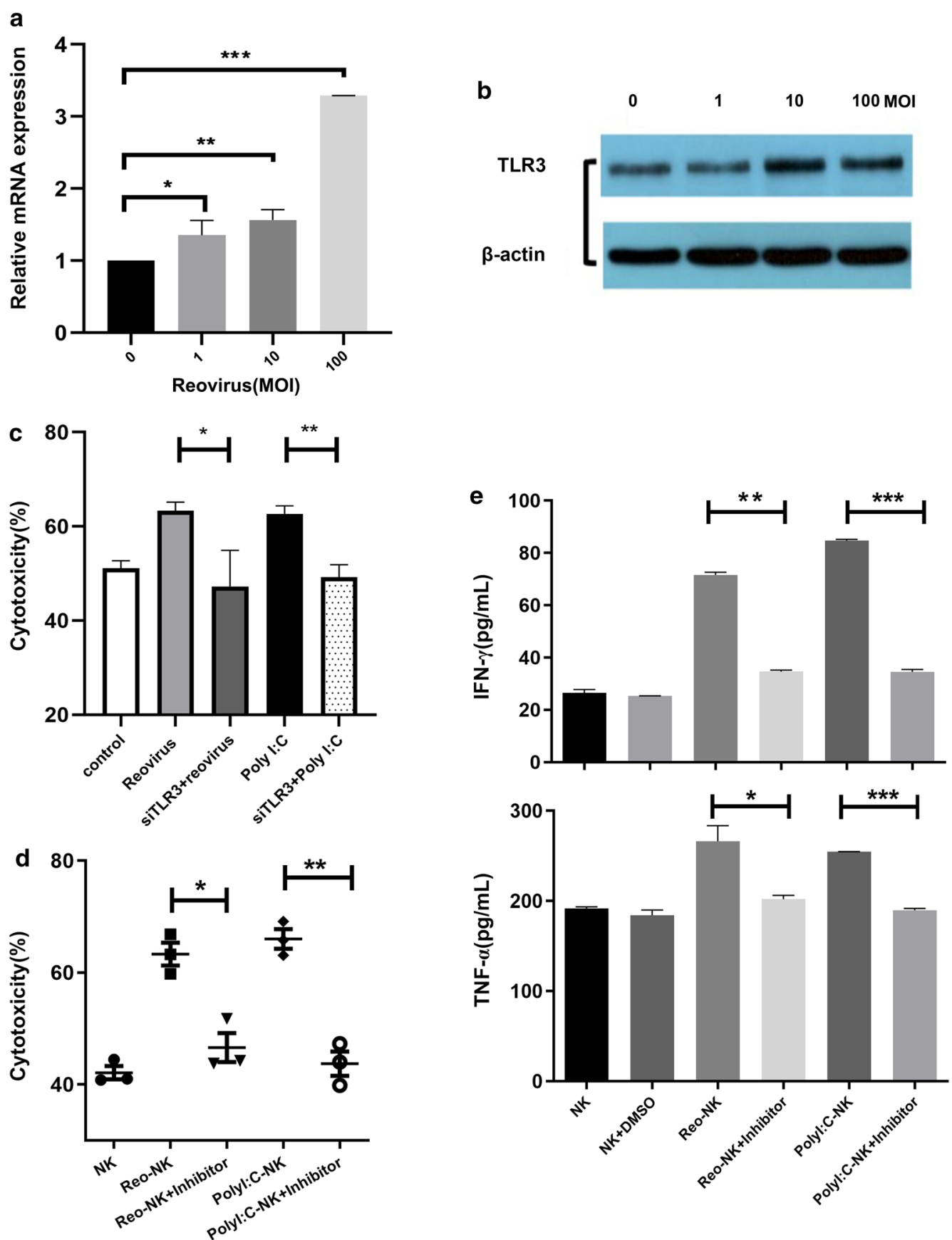

Fig. 5 TLR3 is required for NK cell response to reovirus. NK cells were stimulated with 1, 10, or 100 MOI reovirus for $12 \mathrm{~h}$. Untreated NK cells were used as a control. a Fold changes in TLR3 gene expression of NK cells relative to control cells, measured by qPCR. $\mathbf{b}$ Western blot analysis of TLR3 expression of NK cells. c NK cells were transfected with siTLR3, and knockdown NK cells were then stimulated with $10 \mathrm{MOI}$ reovirus or $1 \mu \mathrm{gg} / \mathrm{mL}$ Poly(l:C) for $12 \mathrm{~h}$. Cytotoxicity of control and TLR3 knockdown NK cells against DLD-1 cells was measured by CCK-8 assay. $\mathbf{d}$ NK cells were activated with $10 \mathrm{MOI}$ reovirus or $1 \mu \mathrm{g} / \mathrm{mL}$ Poly(l:C) in the presence or absence of $10 \mu \mathrm{M}$ TLR3/dsRNA complex inhibitor for $12 \mathrm{~h}$. Cytotoxicity of chemically inhibited and control NK cells against DLD-1 cells was measured by CCK- 8 assay. e TNF- $\alpha$ and IFN- $\gamma$ levels in culture supernatants were quantified by ELISA. Error bars represent means $\pm S D .{ }^{*} p<0.05,{ }^{* *} p<0.01,{ }^{* * *} p<0.001$ 


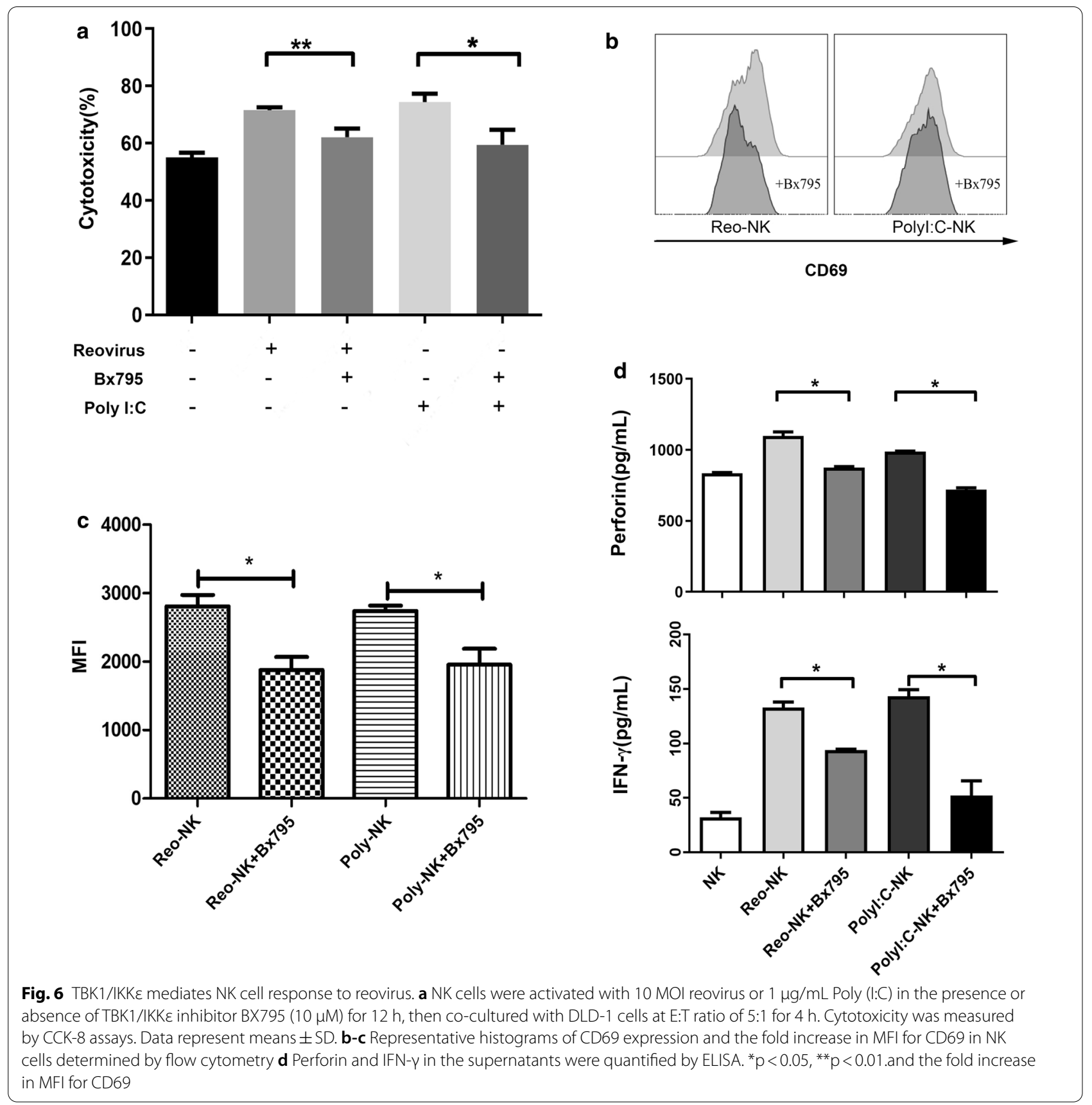

\section{Discussion}

Oncolytic viruses (OV) are a promising class of anti-cancer therapeutics that selectively infect, replicate within, and kill tumor cells. The unique susceptibility of cancer cells to OV infection is the result of defective immune responses and aberrant cellular signaling that accompanies tumorigenesis [27-29]. Following internalization, oncolytic viruses hijack the cell's transcriptional and translational machinery and trigger cell death by a variety of necrotic, apoptotic, and immune-mediated pathways. Reovirus is a nonenveloped dsRNA virus that preferentially replicates in KRAS mutant cells. In light of this interesting and potentially extremely useful property of specifically targeting and killing tumor cells, reovirus has thus come under evaluation as a therapeutic agent in many clinical trials, worldwide [30, 31]. For example, a phase I CRC clinical trial is currently underway to test the efficacy of oncolytic reovirus in combination with chemotherapy (NCT01274624) [32]. In addition to direct oncolysis, recent discoveries have also shown that 
antigens released from reovirus-infected tumor cells are capable of inducing potent anti-tumor immunity, which may be pivotal to the therapeutic effects of the virus [33-35].

In this study, we demonstrate that ex vivo-expanded NK cells can be directly activated by reovirus. NK cells activated by reovirus showed strongly enhanced cytotoxicity against different human CRC cell lines, regardless of differing levels of EGFR expression and KRAS mutation status. In addition, exposure to reovirus led to significant up-regulation of the NK activation marker CD69 as well as transcriptional induction of perforin, granzymes, and TNF. Previous studies revealed that reovirus preferentially replicates in and induces apoptosis of KRAS mutant CRCs, and that cetuximab-mediated ADCC activity is correlated with the expression level of EGFR. Wild-type $K R A S$ or low EGFR expressing tumor cells are therefore likely to exhibit increased resistance to lysis by reovirus or cetuximab-mediated ADCC $[6,36,37]$. In this study, we observed that $K R A S$-mutant and KRAS-WT CRC cell lines showed similar sensitivity to reovirus-activated NK cell killing, and furthermore, tumor cells with both high and low EGFR expression were equally sensitive to cetuximab-induced ADCC, thus overcoming these obstacles to monotherapy. Reovirus activation of NK cells in combination with cetuximab thus provides a versatile strategy to circumvent tumor resistance that can be modified to overcome tumor resistance to other tumor-targeting therapies.

As mentioned above, reovirus is currently under evaluation as an oncolytic agent in numerous clinical trials. Unfortunately, reovirus is ubiquitous in the environment and most adults possess NABs due to prior exposure or following intravenous therapy [38]. Thus, reovirus therapy is seriously curtailed by NABs if administered systemically. However, previous work has revealed that reovirus can be protected from NABs by carriage on PBMCs or DCs, which can also deliver reovirus to the

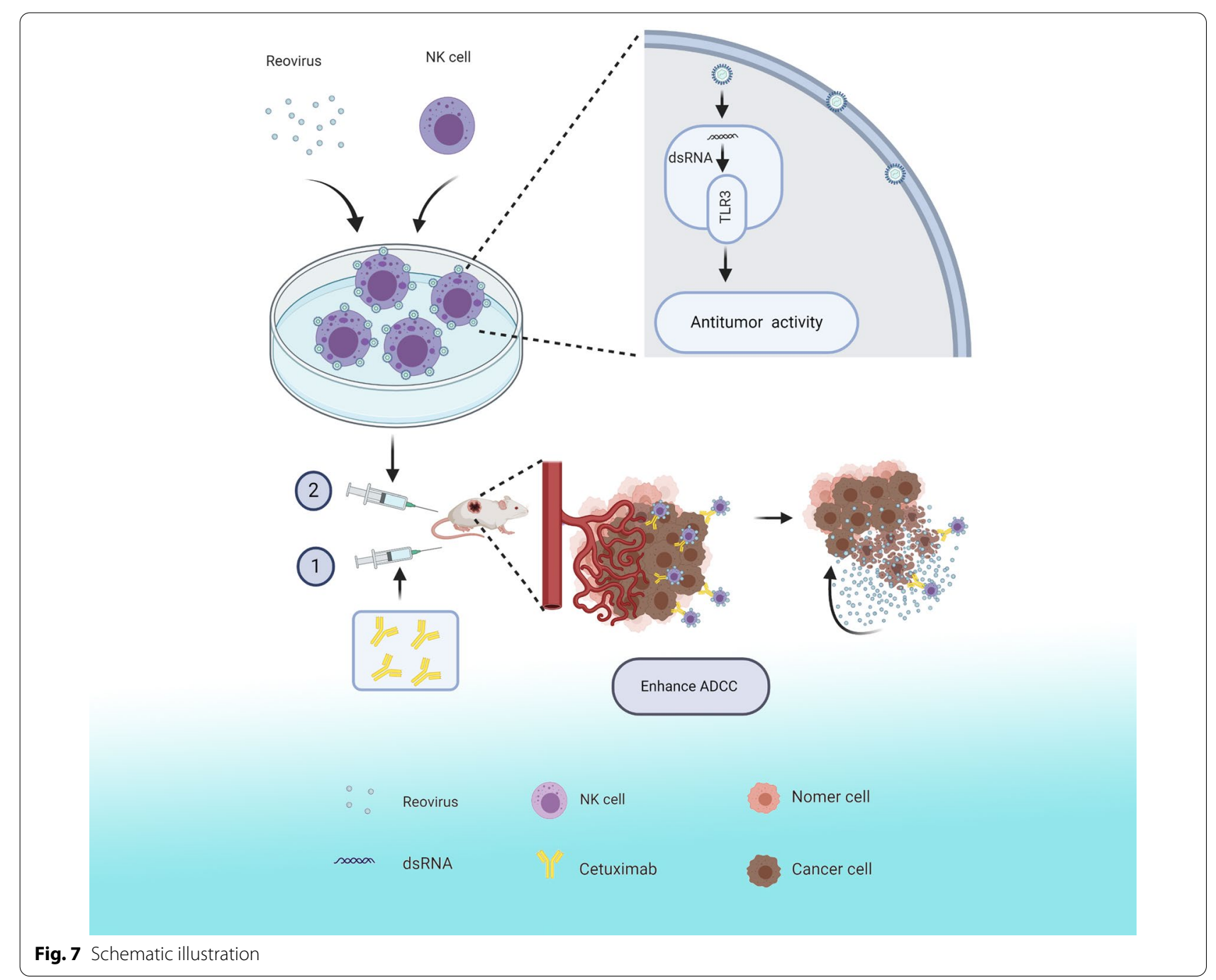


tumor site [39]. Therefore, combination of reovirus with cytotoxic adaptive cell therapy shows considerable promise as an anticancer therapeutic strategy [40, 41]. In previous work, we have shown that cytokine-induced killer (CIK) cells or ex vivo-expanded NK cells can be used as a protective delivery vehicle to carry reovirus to the tumor, avoiding antibody neutralization [21, 42]. Moreover, cytotoxicity of CIK cells was increased after loading with reovirus [43]. In this study, the combination strategy can be summarized as follows(Fig. 7):ex vivo-expanded NK cells were pretreated and loaded with reovirus, which subsequently activated the NK cells and enhanced their cytotoxicity. Our data further show that reovirus-loaded NK cells are capable of depositing active reovirus onto tumor sites when combined with cetuximab. Once in contact with reovirus-loaded NK cells, the cetuximab potentially functions as a bridge to facilitate delivery of the reovirus to the tumor cells.

Although previous in vitro studies have shown that NK cells can be activated by reovirus within PBMCs, resulting in increased NK anti-leukemia activity, which suggests that reovirus in fact activates DCs or monocytes, which in turn activate the NK cells $[44,45]$. Moreover, results from a colorectal cancer clinical trial also suggested that a reovirus-mediated increase in human NK cell activity in vivo was likely due to cross-talk between NK cells and reovirus-activated DCs [46]. This discrepancy with our data is likely due to differences in experimental conditions. First, we used highly purified, fresh, ex vivo-expanded NK cells (>95\%) instead of PBMCs. Second, we stimulated NK cells with different titers of reovirus, and found reovirus stimulation enhanced NK cell cytotoxicity in a dose-dependent manner. Finally, the cytotoxicity of reovirus-activated NK cells was reduced after TLR3 knockdown or blockade of TLR3 signaling pathways and, we presume, the mechanism of TLR3dependent NK cell activation by reovirus.

TLR3 is a pattern recognition receptor that recognizes and responds to dsRNA. We found only moderate levels of TLR3 expression in unstimulated NK cells, in agreement with results reported by Schmidt et al. [24]. The use of TLR3 agonists to enhance the cytotoxicity of NK cells has been well-described [47, 48]. Poly(I:C) is a synthetic dsRNA analog which has been consistently used to induce TLR3 signaling in vitro and in vivo. Previous studies have shown that NK cells can directly recognize and respond to Poly(I:C),which stimulates NK cellmediated cytotoxicity and leads to the up-regulation of TLR3 and activation marker CD69 [24]. NK cells respond to Poly(I:C) by producing IFN- $\gamma$. In this study, we confirmed that the cytotoxicity of NK cells was enhanced by reovirus, and we show that stimulation with reovirus increased TLR3 transcript and protein levels in NK cells.
Furthermore, our results demonstrate that TLR3 knockdown significantly reduced reovirus-activated NK cell cytotoxicity, and that inhibitor of TLR3/dsRNA complex, which prevents dsRNA binding to TLR3, also significantly reduced reovirus activation of NK cell cytotoxicity and cytokine (TNF- $\alpha$ and IFN- $\gamma$ ) release. Consistent with these results, we show that in reovirus-activated NK cells, cytotoxicity, CD69 expression, and perforin and IFN- $\gamma$ secretion were all reduced by treatment with $\mathrm{Bx} 795$, an inhibitor of TBK1/IKKe that acts as a common mediator in the signaling pathways of TLR3. Poly(I:C) was used in these experiments as a positive control. These results thus show that TLR3 is critical for NK cell response to Poly(I:C) and reovirus, and that reovirus promotes the activation of NK cells through a mechanism that is probably similar to that of Poly(I:C).

\section{Conclusions}

In summary, this study provides insight into ex vivoexpanded NK cell activation by reovirus for CRC therapy. We demonstrated that reovirus directly enhances NK cell cytotoxicity against CRC cells in a TLR-3-dependent manner. NK cells act as carriers to transfer the reovirus to the tumor site, and the combination of cetuximab and reovirus-activated NK cells enhances the ADCC effect. As a result, reovirus can directly lyse tumor cells at the tumor site, while reovirus activated-NK cells enhance the ADCC effect to kill tumors, thus providing a framework for further development of effective, clinical strategies against CRC.

\section{Abbreviations}

CRC: Colorectal cancer; NABs: Neuralizing antibodies; EGFR: Epidermal growth factor receptor; ADCC: Antibody-dependent cell-mediated cytotoxicity; DCs: Dendritic cells; NK: Natural killer; TLR3: Toll-like receptor 3; Poly (I:C): Polyinosinic:polycytidylic acid; PBMCs: Peripheral blood mononuclear cells; CCK-8: Cell Counting Kit-8; dsRNA: Double stranded RNA; MOI: Multiplicity of infection; PFU: Plaque-forming unit; ACT: Adoptive cell therapy; CIK: Cytokineinduced killer.

\section{Supplementary Information}

The online version contains supplementary material available at https://doi. org/10.1186/s12967-021-02853-y.

Additional file 1: Table S1. The PCR primers used in this study. Additional file 2: Figure S1. Expression of intracellular TLR3 in reovirustreated NK cells.

Additional file 3: Figure S2. Western blots showing TLR3 expression in NK cells transfected with siTLR3.

\section{Acknowledgements}

Not applicable.

\section{Authors' contributions}

$X Z$ conceived and designed the study. ZXH revised the manuscript. SQL designed experiments and analyzed data. YZG. supervised the mice 
experiment and wrote the manuscript. YYA, XJL, XQC, XYW, CXL, performed the majority of the experiments and interpreted the data. WWOY and NXW assisted with literature searches and appraised relevant studies. All authors read and approved the final manuscript.

\section{Funding}

This research was funded by the National Natural Science Foundation of China (No. 81860542), funded by the Guizhou Provincial Natural Science Foundation [Grant No. (2019)5663], the Program for Top Scientific and Technological Talents in Guizhou Province [Grant No. KY (2018)049], and funded by Key Program for Science and Technology of Guizhou Province [Grant No. ZK(2021)012].

\section{Availability of data and materials}

The datasets analyzed during the current study are available from the corresponding author on reasonable request.

\section{Declarations}

\section{Ethics approval and consent to participate}

All procedures performed in studies involving animals were approved by the Ethics Committee of Guizhou Medical University (Ref: 2000803).

\section{Consent for publication}

Not applicable.

\section{Competing interests}

The authors declare that they have no competing interests.

\section{Author details}

${ }^{1}$ Center for Stem Cell and Tissue Engineering Research/School of Basic Medical Sciences, Guizhou Medical University, Guiyang 550004, Guizhou, China. ${ }^{2}$ Key Laboratory of Adult Stem Cell Transformation Research, Guiyang 550004, China. ${ }^{3}$ State Key Laboratory of Biotherapy and Cancer Center, West China Hospital, Sichuan University, and Collaborative Innovation Center for Biotherapy, Chengdu 610041, China. ${ }^{4}$ Department of Oncology, The Affiliated Hospital of Guizhou Medical University and Guizhou Cancer Hospital, Guiyang 550004, China. ${ }^{5}$ Department of Pediatrics, Affiliated Hospital of Zunyi Medical University, Zunyi 563000, Guizhou, China.

Received: 31 January 2021 Accepted: 21 April 2021

Published online: 01 May 2021

\section{References}

1. Siegel RL, Miller KD, Goding-Sauer A, et al. Colorectal cancer statistics, 2020. CA. 2020;70(3):145-64.

2. Keum N, Giovannucci E. Global burden of colorectal cancer: emerging trends, risk factors and prevention strategies. Nat Rev Gastroenterol Hepatol. 2019;16(12):713-32.

3. Xie YH, Chen YX, Fang JY. Comprehensive review of targeted therapy for colorectal cancer. Signal Transduct Target Ther. 2020;5(1):22.

4. Guren TK, Thomsen M, Kure EH, et al. Cetuximab in treatment of metastatic colorectal cancer: final survival analyses and extended RAS data from the NORDIC-VII study. Br J Cancer. 2017;116(10):1271-8.

5. Heinemann V, von Weikersthal LF, Decker T, et al. FOLFIRI plus cetuximab or bevacizumab for advanced colorectal cancer: final survival and per-protocol analysis of FIRE-3, a randomised clinical trial. $\mathrm{Br} J$ Cancer. 2020;124(3):587-94

6. Seo Y, Ishii Y, Ochiai $H$, et al. Cetuximab-mediated ADCC activity is correlated with the cell surface expression level of EGFR but not with the KRAS/BRAF mutational status in colorectal cancer. Oncol Rep. 2014;31(5):2115-22.

7. Taylor RJ, Saloura V, Jain A, et al. Ex vivo antibody-dependent cellular cytotoxicity inducibility predicts efficacy of cetuximab. Cancer Immunol Res. 2015;3(5):567-74.

8. Lo Nigro C, Macagno M, Sangiolo D, et al. NK-mediated antibodydependent cell-mediated cytotoxicity in solid tumors: biological evidence and clinical perspectives. Ann Transl Med. 2019;7(5):105-105.
9. Zahavi D, AIDeghaither D, O'Connell A, et al. Enhancing antibodydependent cell-mediated cytotoxicity: a strategy for improving antibodybased immunotherapy. Antibody Ther. 2018;1 (1):7-12.

10. Kohrt HE, Colevas AD, Houot R, et al. Targeting CD137 enhances the efficacy of cetuximab. J Clin Invest. 2014;124(6):2668-82.

11. Jewett A, Kos J, Kaur K, et al. Natural killer cells: diverse functions in tumor immunity and defects in pre-neoplastic and neoplastic stages of tumorigenesis. Mol Ther. 2020;16:41-52.

12. Guan Y, Chambers CB, Tabatabai T, et al. Renal cell tumors convert natural killer cells to a proangiogenic phenotype. Oncotarget. 2020;11(26):2571-85.

13. Melaiu O, Lucarini V, Cifaldi L, et al. Influence of the Tumor Microenvironment on NK cell function in solid tumors. Front Immunol. 2020;10:3038-3038.

14. Zhang C, Liu Y. Targeting NK Cell Checkpoint Receptors or Molecules for Cancer Immunotherapy. Front Immunol. 2020;11:1295-1295.

15. da Silva IP, Gallois A, Jimenez-Baranda S, et al. Reversal of NK-cell exhaustion in advanced melanoma by Tim-3 blockade. Cancer Immunol Res. 2014;2(5):410-22.

16. Liu Y, Cheng $Y, X u Y$, et al. Increased expression of programmed cell death protein 1 on NK cells inhibits NK-cell-mediated anti-tumor function and indicates poor prognosis in digestive cancers. Oncogene. 2017;36(44):6143-53.

17. Freund-Brown J, Chirino L, Kambayashi T. Strategies to enhance NK cell function for the treatment of tumors and infections. Crit Rev Immunol. 2018;38(2):105-30.

18. Schmidt L, Eskiocak B, Kohn R, et al. Enhanced adaptive immune responses in lung adenocarcinoma through natural killer cell stimulation. Proc Natl Acad Sci. 2019;116(35):17460-9.

19. Rajasekaran N, Chester C, Yonezawa A, et al. Enhancement of antibodydependent cell mediated cytotoxicity: a new era in cancer treatment. Immunotargets Ther. 2015;4:91-100.

20. Zhao X, Rajasekaran N, Chester C, et al. Natural killer cells activated by oncolytic reovirus enhance cetuximab mediated antibody dependent cellular cytotoxicity in an in vitro and in vivo model of colorectal cancer. Blood. 2015;126(23):3439-3439.

21. Xiaoqing C, Nianxue W, Shiqi L, et al. Cytotoxic effect of in vitro expanded NK cell-carrying oncolytic reovirus on colorectal cancer cells. Chin J Cancer Biother. 2019;26(5):492-9.

22. Zhang M, Wen B, Anton OM, et al. IL-15 enhanced antibody-dependent cellular cytotoxicity mediated by NK cells and macrophages. Proc Natl Acad Sci. 2018;115(46):E10915-24.

23. Sivori S, Falco M, Chiesa MD, et al. CpG and double-stranded RNA trigger human NK cells by Toll-like receptors: Induction of cytokine release and cytotoxicity against tumors and dendritic cells. Proc Natl Acad Sci USA. 2004;101(27):10116-21.

24. Schmidt KN, Leung B, Kwong M, et al. APC-independent activation of NK cells by the toll-like receptor 3 agonist double-stranded RNA. J Immunol. 2004;172(1):138.

25. Matsumoto M, Tatematsu M, Nishikawa F, et al. Defined TLR3-specific adjuvant that induces NK and CTL activation without significant cytokine production in vivo. Nat Commun. 2015;6(1):6280.

26. Veluchamy JP, Spanholtz J, Tordoir M, et al. Combination of NK cells and cetuximab to enhance anti-tumor responses in RAS mutant metastatic colorectal cancer. PLoS ONE. 2016;11(6):e0157830-e0157830.

27. Struzik J, Szulc-Dąbrowska L. NF-kB signaling in targeting tumor cells by oncolytic viruses-therapeutic perspectives. Cancers. 2018;10(11):426.

28. Fernandes J. Oncogenes: the passport for viral oncolysis through PKR Inhibition. Biomark Cancer. 2016;8:101-10.

29. Zhao X, Chester C, Rajasekaran N, et al. Strategic combinations: the future of oncolytic virotherapy with reovirus. Mol Cancer Ther. 2016;15(5):767-73.

30. Samson A, Bentham MJ, Scott K, et al. Oncolytic reovirus as a combined antiviral and anti-tumour agent for the treatment of liver cancer. Gut. 2018;67(3):562-73.

31. Müller L, Berkeley R, Barr T, et al. Past, present and future of oncolytic reovirus. Cancers (Basel). 2020;12:11.

32. Parakrama R, Chaudhary I, Coffey MC, et al. Immune response to reovirus (REO) in phase I study with chemotherapy in patients with KRAS mutant metastatic colorectal cancer (mCRC). J Clin Oncol. 2018;36(5_suppl):217-217. 
33. Prestwich RJ, Errington F, llett EJ, et al. Tumor infection by oncolytic reovirus primes adaptive antitumor immunity. Clin Cancer Res. 2008;14(22):7358-66.

34. Russell L, Peng KW, Russell SJ, et al. Oncolytic viruses: priming time for cancer immunotherapy. BioDrugs. 2019;33(5):485-501.

35. Davola ME, Mossman KL. Oncolytic viruses: how "lytic" must they be for therapeutic efficacy? Oncolmmunology. 2019;8(6):e1581528.

36. Maitra R, Seetharam R, Tesfa $L$, et al. Oncolytic reovirus preferentially induces apoptosis in KRAS mutant colorectal cancer cells, and synergizes with irinotecan. Oncotarget. 2014;5(9):2807-19.

37. Jiffry J, Thavornwatanayong T, Rao D, et al. Oncolytic reovirus (pelareorep) induces autophagy in KRAS-mutated colorectal cancer. Clin Cancer Res. 2020;27(3):865-76.

38. Berkeley RA, Steele LP, Mulder AA, et al. Antibody-neutralized reovirus is effective in oncolytic virotherapy. Cancer Immunol Res. 2018;6(10):1161-73.

39. Adair RA, Roulstone V, Scott KJ, et al. Cell carriage, delivery, and selective replication of an oncolytic virus in tumor in patients. Sci Transl Med. 2012;4(138):138ra77.

40. Ilett EJ, Bárcena M, Errington-Mais F, et al. Internalization of oncolytic reovirus by human dendritic cell carriers protects the virus from neutralization. Clin Cancer Res. 2011;17(9):2767-76.

41. Jennings VA, llett EJ, Scott KJ, et al. Lymphokine-activated killer and dendritic cell carriage enhances oncolytic reovirus therapy for ovarian cancer by overcoming antibody neutralization in ascites. Int J Cancer. 2014;134(5):1091-101.
42. Zhao X, Ouyang W, Chester C, et al. Cytokine-induced killer cell delivery enhances the antitumor activity of oncolytic reovirus. PLOS ONE. 2017:12(9):e0184816.

43. Zhao X, Rajasekaran N, Chester C, et al. Reovirus activated NK cells show enhanced cetuximab mediated antibody-dependent cellular cytotoxicity against colorectal cancer cells. J Immunother Cancer. 2015;3(Suppl 2):P340-P340.

44. Parrish C, Scott GB, Migneco G, et al. Oncolytic reovirus enhances rituximab-mediated antibody-dependent cellular cytotoxicity against chronic lymphocytic leukaemia. Leukemia. 2015;29(9):1799-810.

45. Hall K, Scott KJ, Rose A, et al. Reovirus-mediated cytotoxicity and enhancement of innate immune responses against acute myeloid leukemia. Bioresour Open Access. 2012;1(1):3-15.

46. El-Sherbiny YM, Holmes TD, Wetherill LF, et al. Controlled infection with a therapeutic virus defines the activation kinetics of human natural killer cells in vivo. Clin Exp Immunol. 2015;180(1):98-107.

47. Kaczanowska S, Joseph AM, Davila E. TLR agonists: our best frenemy in cancer immunotherapy. J Leukoc Biol. 2013;93(6):847-63.

48. Sultan H, Wu J, Fesenkova VI, et al. Poly-IC enhances the effectiveness of cancer immunotherapy by promoting T cell tumor infiltration. J Immunother Cancer. 2020;8(2):e001224.

\section{Publisher's Note}

Springer Nature remains neutral with regard to jurisdictional claims in published maps and institutional affiliations.
Ready to submit your research? Choose BMC and benefit from:

- fast, convenient online submission

- thorough peer review by experienced researchers in your field

- rapid publication on acceptance

- support for research data, including large and complex data types

- gold Open Access which fosters wider collaboration and increased citations

- maximum visibility for your research: over 100M website views per year

At BMC, research is always in progress.

Learn more biomedcentral.com/submissions 